\title{
Temporal height properties of the exploding granules
}

\author{
Th. Roudier ${ }^{1}$, M. T. Eibe ${ }^{2}$,J. M. Malherbe ${ }^{2}$, M. Rieutord ${ }^{1,4}$, P. Mein ${ }^{2}$, N. Mein ${ }^{2}$, and M. Faurobert ${ }^{3}$ \\ 1 Laboratoire d'Astrophysique de Toulouse, Observatoire Midi-Pyrénées, 14 avenue E. Belin, 31400 Toulouse, \\ France \\ 2 DASOP, Observatoire de Paris, Section de Meudon, 92195 Meudon, France \\ 3 Observatoire de la Cote d'Azur, BP 4229, 06304 Nice Cedex 04, France \\ 4 Institut Universitaire de France, France
}

Received 26 October 2000 / Accepted 22 December 2000

\begin{abstract}
Based on time series of 2D MSDP spectrograms, taken at the Turret Dome in Pic du Midi, we present the temporal evolution of exploding granules in intensity and Doppler velocity through the solar photosphere. We describe the penetration of exploding granules in the solar photosphere during their lifes and the related phenomena like the "Bright Plumes" located in the downflowing plasma just on the edge of the granule. We suggest a possible scenario of the exploding granule evolution in the solar photosphere.
\end{abstract}

Key words. Sun: chromosphere - Sun: photosphere

\section{Introduction}

Nowadays, there is general agreement about the flow organization at the granular scale on the solar surface which is in great part controled by the relative adjustment of the granules strength. Indeed, the expansion of granules is due to a pressure excess relative to neighbouring granules (Ploner et al. 1999). In the simulations, granules evolve by pushing out against and being pushed in by their neighbouring granules, and by being split by overlying fluid that cools and sinks (Stein \& Nordlund 1998).

Recently, the important role played by the Strong Positive Divergences (SPDs) in the formation of photospheric network has been put into light by Rieutord et al. (2000). The exploding granules constitute the highest amplitude part of the SPDs and thus much contribute to structure the surface horizontal flow fields which build the large-scale organisation of the discrete magnetic field (i.e. flux tubes). The competition between SPDs with different amplitudes and their recurrence in neighbouring locations, is one of the solutions proposed to explain the large-scale organisation of floating corks similar to the photospheric network (Rieutord et al. 2000).

The exploding granules have been known to be one of the most dynamical features of the sun's photosphere. This kind of granules have attracted the attention of many workers, since their discovery by Rösch and colleagues (Carlier et al. 1968): for instance on space SOUP data (Title et al. 1989) and more recently on very high

Send offprint requests to: Th. Roudier,

e-mail: thierry.roudier@obs-mip.fr resolution La Palma data (Hirzberger et al. 1999). From white light observations, the morphological and temporal evolutions are now quite well described (see references in Hirzberger et al. 1999). But, although the knowledge of the exploding granules at the bottom of the photosphere is quite precise, their temporal evolution throughout the photosphere is not known. This is due, in part, to the difficulty of getting time series of two-dimensional highresolution spectrographic data.

Thanks to a time sequence (16 min) of MSDP spectra observation in $\mathrm{NaD}_{2}$ line, we shall describe, in this paper, the temporal evolution of 9 exploding granules in intensity and Doppler velocity throughout the photosphere. Some unexpected relations are observed between the exploding granule intensity located in the bottom of the photosphere and the bright plumes located at the top of the photosphere in the downflow (see Espagnet et al. 1995) on the edge of the exploding granule. The interaction visible in the intensity field between the exploding granules and the plumes seems to be related to the time of the explosion.

\section{Observations and reduction}

A series of $642 \mathrm{D}$ spectrograms was taken at the Turret Dome, with the $50 \mathrm{~cm}$ refractor of the Pic du Midi Observatory on October 22, 1988, using the MSDP spectrograph at $\lambda=5890 \AA$ ( $\mathrm{NaD}_{2}$ line), under excellent seeing conditions. However, because of the relatively long exposure time $(0.54 \mathrm{~s})$ on photographic film, the mean spatial resolution is around $0.5^{\prime \prime}$. The time interval between 
successive frames is $15 \mathrm{~s}$ and the duration of the sequence is $16 \mathrm{~min}$.

The digitized spectrograms cover a quiet area of the sun of $185^{\prime \prime} \times 17^{\prime \prime}\left(1072 \times 101\right.$ pixels, pixel size $\left.=0.168^{\prime \prime}\right)$. The reduction of this data has been extensively described in Roudier et al. (1991) and Espagnet et al. (1995), in particular the computation of the intensity and Doppler velocity fluctuations at different depths in the $\mathrm{NaD}_{2}$ line. The 64 images have been aligned by computing the interspectra between consecutive intensity frames and adjusting to zero the phase spectra. The accuracy of the alignments is better than one pixel. After spectrogram processing and image alignment, we are left with a field of view of $172^{\prime \prime} \times 8^{\prime \prime}$. Every frame of the time series has also been filtered from the 5-mn oscillations, by applying a "subsonic filter" in the $k-\omega$ space which removes all the structures for which $\omega>v_{\varphi} k$ with the velocity phase $v_{\varphi}$ set to $6 \mathrm{~km} \mathrm{~s}^{-1}$, below the sound velocity at this altitude (Espagnet et al. 1995). The final data consist of 11 filtered times series of the intensity pattern (at ten levels in the photosphere plus the continuum) and 10 time series of the vertical velocity pattern. The size of the smallest elements visible in the observation is about $0.7^{\prime \prime}$. Velocities are derived from the dopplershift at different heights in the profiles using a classical lambdameter technique.

\section{Intensity and Doppler velocity through the photosphere}

One of the observer's dream is to draw the velocity and temperature fields at each point of the photosphere like theoretician do in their simulations i.e. Stein \& Nordlund (1999), http://ww.pa.msu.edu/ ${ }^{\text {steinr/images/granule.gif }}$

However, the interpretation of the observed data is rendered difficult because of three complications (Topka \& Title 1991):

- finite resolution and seeing distortion;

- 5-mn oscillations contribute very significantly to the velocity field in this part of the solar atmosphere;

- the measurements, using Doppler shifts, do not originate from a unique height in the atmosphere, but from contributions of a range of heights.

In our case the quality of the data of the MSDP sequence, namely the good spatial, temporal and spectral resolution, helped us to minimize these inconveniences: in particular the simultaneous two dimensional fields in intensity (i.e. temperature) and velocity fluctuations allow us to take out the $5 \mathrm{mn}$ oscillations. Hence, the temporal evolution of the exploding granules through the photosphere can be analysed quite well by restricting our study to three levels: low $(0-150 \mathrm{~km})$, middle $(150-300 \mathrm{~km})$ and high (300-600 km) photosphere.

Nine exploding granules were identified and followed during their lifetime through the photosphere during the 16 minutes of our sequence. Although the statistical sample of exploding granules is weak, this time sequence filtered in $k-\omega$ space of the 5 min oscillation, gives a unique
Table 1. RFT and RFV barycenters in log $\mathrm{m}$ and height $(\mathrm{km})$ coordinates

\begin{tabular}{ccccc}
\hline$\Delta \lambda(\AA)$ & RFT log $m$ & $\begin{array}{c}\text { Barycenter } \\
\text { Height }(\mathrm{km})\end{array}$ & RFV log $m$ & $\begin{array}{c}\text { Barycenter } \\
\text { Height }(\mathrm{km})\end{array}$ \\
\hline 0.072 & -0.34 & 267 & -2.05 & 657 \\
\hline 0.144 & -0.08 & 202 & -0.60 & 327 \\
\hline 0.288 & 0.19 & 131 & 0.05 & 170 \\
\hline 0.324 & 0.23 & 120 & 0.09 & 160 \\
\hline 0.432 & 0.31 & 98 & 0.16 & 141 \\
\hline
\end{tabular}

opportunity to learn simultaneously on the Doppler velocity and intensity field at very high spatial and temporal resolutions. With the limitation quoted above, it has been possible to extract some common phenomena to these exploding granules.

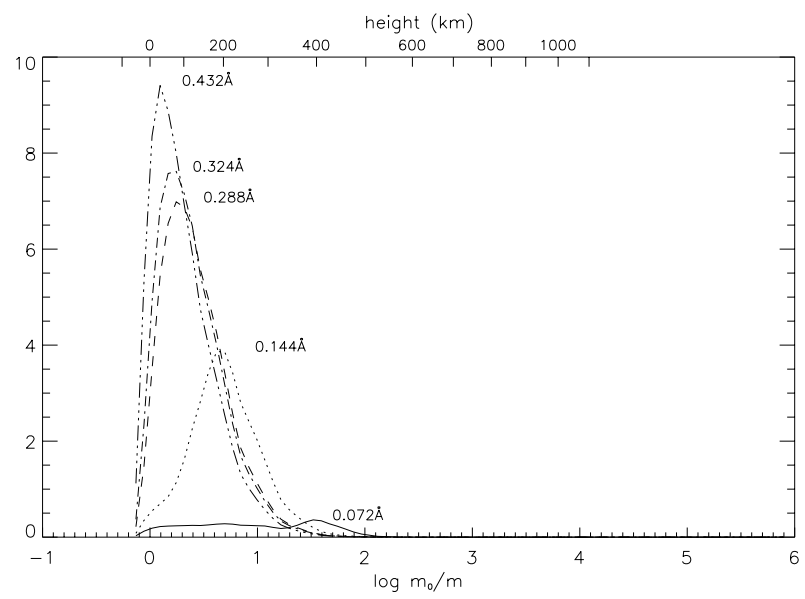

Fig. 1. Temperature Reponse Functions (RFTs) (adimensional unit) at different wavelengths in $\AA$. The $x$-axis gives the photosphere height and the $\log \left(m_{0} / m\right)$ unit

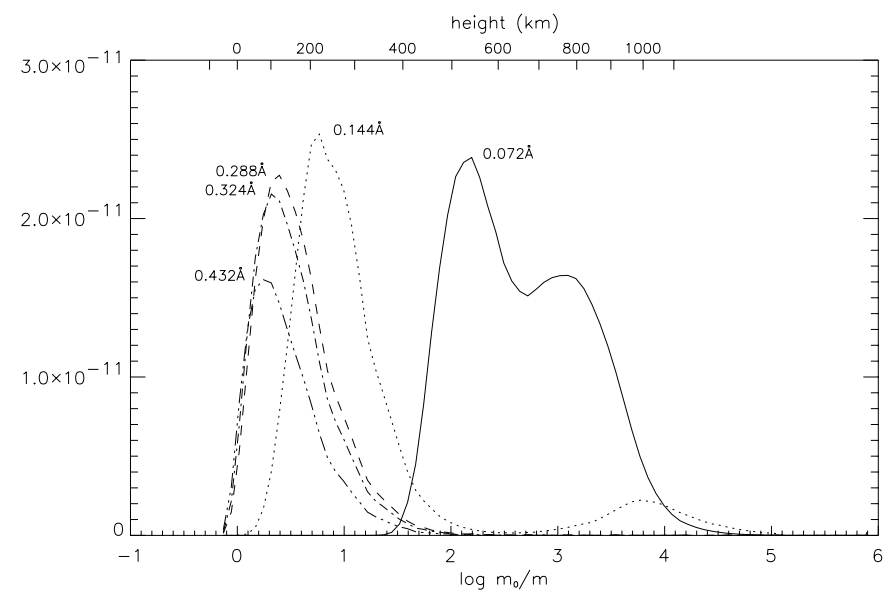

Fig. 2. Velocity Reponse Functions (RFVs) $\left(\mathrm{erg} \mathrm{cm}^{-3} \mathrm{ster}^{-1}\right.$ $\left.\mathrm{Hz}^{-1}\right)$ at different wavelengths in $\AA$. The $x$-axis gives the photosphere height and the $\log \left(m_{0} / m\right)$ unit 
We have calculated response functions of the $\mathrm{NaD}_{2}$ line to temperature and velocity perturbations in the atmosphere (Eibe et al. 2000). Perturbations were simulated numerically as small-amplitude disturbances in the reference local values of temperature and velocity at each height, as adopted from the VAL C mean quiet-sun model Vernazza et al. (1981). In the case of temperature, relative perturbations $\delta T / T$ were considered whereas for velocity we used absolute perturbations, $\delta V$. The subsequent fluctuations of the emergent line intensity were calculated by using the non-LTE code MULTI (Carlsson 1986). Figures 1 and 2 show temperature and velocity response functions for selected wavelengths, allowing us to probe the photosphere at the three different levels (see Table 1):

- $\Delta \lambda=0.432,0.324,0.288 \AA$ low photosphere;

$-\Delta \lambda=0.144 \AA$ middle photosphere;

$-\Delta \lambda=0.072 \AA$ high photosphere.

These wavelengths are used below in Figs. 4, 5, 7, 9, for the plot of the intensity and Doppler velocity at the centre of the exploding granules and in the centre of the Bright plumes.

The barycentres of the response functions (in velocity and temperature) at those particular wavelengths are given in Table 1. For the velocity response functions, it appears that contributions from large heights $\left(\log m / m_{0}<\right.$ -2 ) is not always negligible as opposed to the case of temperature. This conditions barycentre calculations. " $m$ " stands for the column mass and $\mathrm{m}_{0}$ the column mass at $\tau=5000 \AA$.

\section{Temporal evolution of exploding granules}

To describe the temporal evolution of a typical exploding granule through the photosphere resulting from our analysis, we use as an example the first exploding granule of our selection. In the following, general comments are given to describe the different behaviours of the other exploding granules of our sample. To summarize the characteristic properties of the exploding granules evolution in the 4 dimensions of our data set i.e. $\operatorname{space}(x, y)$, time $(t)$, height $(h)$ and 2 parameters Intensity $(I)$, Doppler Velocity $(V)$, we first plot the 2D temporal evolution, in the middle photosphere, of the intensity and velocity fields. The temporal evolution in "altitude" is illustrated by slices, in $x$ direction centreed onto the exploding granule, in intensity and Doppler velocity computed at different depths in the line.

\section{1. $2 D$ intensity and velocity temporal evolution in the middle photosphere}

In Fig. 3 the exploding granule can be followed, in alternately rows, in the middle photosphere in intensity $(0.144 \AA$ corresponding to a barycentre at $202 \mathrm{~km})$ and Doppler velocity $(0.288 \AA$ corresponding to a barycentre $170 \mathrm{~km}$ ). The two-dimensional granule Doppler velocity, in this domain of altitude, is quite similar to the one found at the bottom of the photosphere. At the location of the exploding granule (see arrows), the temperature reversal is clearly visible in the intensity field.

This temperature reversal is still found above the two elements resulting from the explosion visible at the end of the time sequence. In the rest of our exploding granule sampling, the intensity reversal is only observed above the most vigourous elements in Doppler velocity $\left(v>0.4 \mathrm{~km} \mathrm{~s}^{-1}\right)$ resulting from the explosion.

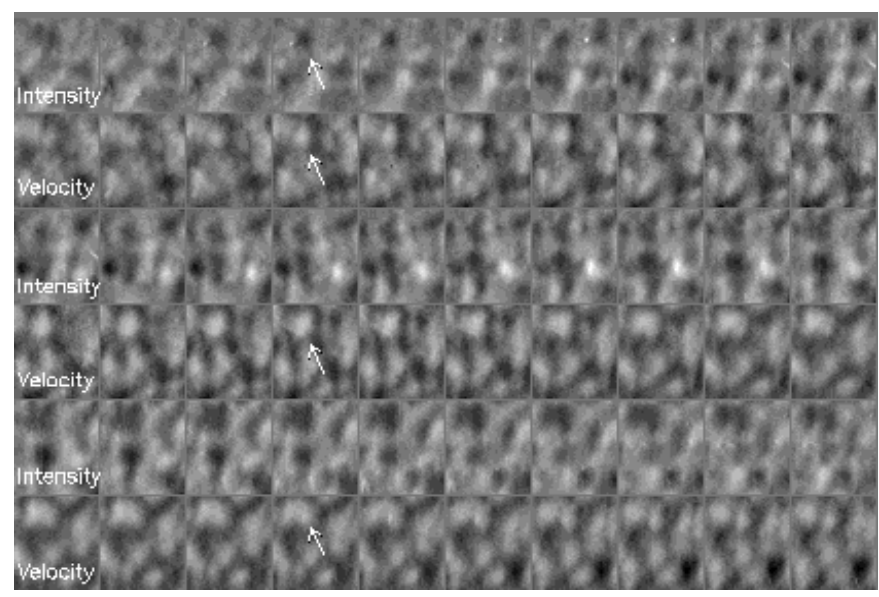

Fig. 3. Time sequence in the middle photosphere, in alternately rows, of the intensity $(0.144 \AA$ corresponding to a barycentre at $202 \mathrm{~km})$ and the Doppler velocity $(0.288 \AA$ corresponding to a barycentre $172 \mathrm{~km})$. At the location of the upward velocities (white) the granule intensity is reversed in the middle photosphere shown here. The time step between two successive frames is $15 \mathrm{~s}$. The field of view of each frame is $6.9^{\prime \prime} \times 7.7^{\prime \prime}$. The extrema of the Doppler velocities in this figure are -0.7 and $0.6 \mathrm{~km} \mathrm{~s}^{-1}$. The arrows indicate the location of the exploding granule. We clearly see from this sequence a bright intensity "ring" with different patches located in the downflowing plasma on the edge of the exploding granule. The brighter intensity patches correspond to the highest downflows (see movie at http://mesola.obspm.fr/gallery/nad2.mpg)

In Fig. 3, around the exploding granule, in the negative Doppler velocities (intergranule), one notes something like a bright "ring" (or patches around granule) in the intensity field. The brightest "patches" are related to the maximum velocity downflow $\left(v<-0.5 \mathrm{~km} \mathrm{~s}^{-1}\right)$.

The brightness of this "ring" increases up to the explosion time and then decreases. The brightness isotropy around exploding granules is more or less conspicuous in our sample of exploding granules. For some of them, there is only a bright patch (in intensity field) on one side where the downflow is more vigourous. These brightness in the middle and high photosphere around exploding granules are identical to the "Bright plumes" described in Espagnet et al. (1995). 


\subsection{Intensity temporal evolution through the photosphere}

The temporal behaviour with altitude of the exploding granules is analysed through the evolution of the "altitude" slices in intensity and Doppler velocity.

In order to take into account of the 3-dimensions of our data $(x, y, h)$, different $(x, h)$ slices in the $y$ direction through the exploding granule have been inspected during our study. The temporal step between two successive slices is $15 \mathrm{~s}$. In this work, ten positions in the line have been selected and characterized by the distance $\Delta \lambda$ from the line centre. The values of the $\Delta \lambda$ used for the photospheric slices are, from the continuum to the core of the line, in $\AA$ : $0.432,0.360,0.324,0.288,0.252,0.216,0.180,0.144$, $0.108,0.072$.

In the intensity slice centreed onto the exploding granule (Fig. 4) we observe, during the first two minutes, the birth of the granule in the low photosphere followed, two minutes later, by the penetration of this intensity up to the bottom of the middle photosphere $(150 \mathrm{~km})$. The maximun of intensity, in the low photosphere, is reached around $5.5 \mathrm{mn}$ after the birth. The temperature reversal above the exploding granule is clearly visible since the beginning, from the top of the middle photosphere $(300 \mathrm{~km})$ to the high photosphere.

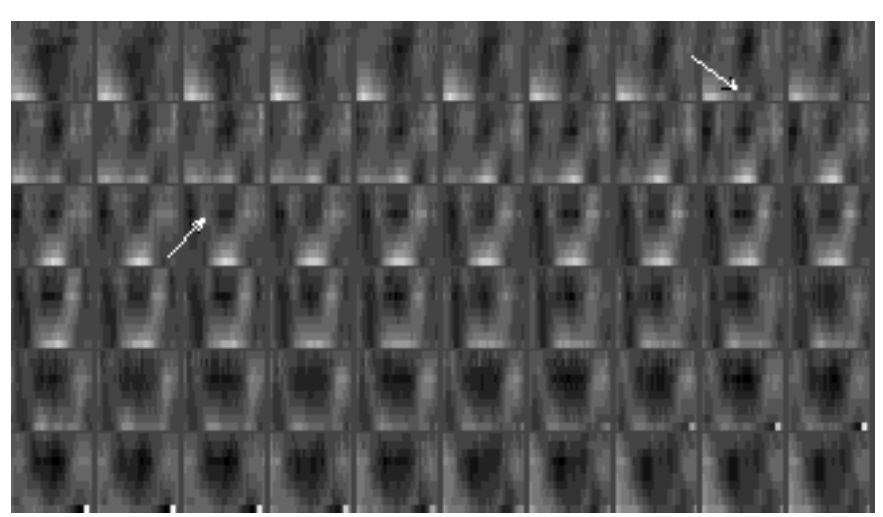

Fig. 4. Time sequence of brightness map in a vertical crosssection (slice) of the photosphere. White areas correspond to the positive intensity. The starting image of the sequence is located in the upper-left corner and the time evolution runs from the left to the right. The field of view in the $x$ direction is $5^{\prime \prime}$. The time step between two consecutive slices is $15 \mathrm{~s}$. The arrow in the upper-right shows the location of the exploding granule in the slice. The arrow in the left-middle part indicates the location of the associated bright plumes. Because the vertical scale has been magnified by a factor 9 with respect to the horizontal scale, the inclination are in reality found between 30 and 40 degres relatively to the horizontal axis (see movie at http://mesola.obspm.fr/gallery/iv.mpg)

Around $1.5 \mathrm{mn}$ after the birth of the exploding granule, a "Bright Plume" appears on the edges of the exploding granule in the high photosphere. This new feature with a size lying between $0.7^{\prime \prime}$ and $1.0^{\prime \prime}$, does not have a counterpart in the low photosphere (see Fig. 4).
Then, during the following $5 \mathrm{~min}$, the Bright Plume propagates down to the middle photosphere. These Bright Plumes are located in the downflows and stay during this phase just on the edge of the exploding granules. Later, a "connection" is observed between these Bright Plumes, located in the top and middle photosphere, and the intensity of the exploding granule in the bottom of the photosphere. The mean time between the birth of the exploding granules and the "connection phase", is around $6 \mathrm{mn}$. In our sample of 9 exploding granules, only 6 of them show an intensity "connection" phase followed by an explosion; another case exhibits an explosion followed by an intensity connection, and the last two cases show only an explosion without intensity "connection".

In the example shown here, the granule explosion happens 3 min after than the intensity "connection". In our sample of exploding granules, this value is found generally around $1 \mathrm{~min}$. The division of the granule due to the explosion in the low photosphere is clearly visible in intensity. The horizontal proper motions of the elements resulting from the explosion are measured between 0.6 and $2 \mathrm{~km} \mathrm{~s}^{-1}$, in good agreement with previous measurements Brandt et al. (1991). The maximun of intensity of the Bright Plumes during their life lies between 20 to 40 percents of the maximun of the intensity of the exploding granule in the low photopshere.

The intensity "connection" between the plume and granule persists up to the death of the granule in all the cases of our sample. These intensity "connection" is generally found tilted relatively to the radial direction. Some tilted intensity field through the photosphere is also reported by Karpinsky (1989); this is probably related to this phenomenon. This may affect the statistical analysis when one measures the Coherence and the Phase coefficients through the photosphere like those computed by different authors (Nesis et al. 1988; Wiehr \& Kneer 1988). These tilts reduce these coefficients when they are computed relatively to the bottom of the photosphere.

\subsection{Velocity temporal evolution through the photosphere}

In the Doppler velocity slices, Fig. 5, the upward Doppler velocity (white) in the exploding granule appears in the low and middle photosphere in the first minutes after the birth of the granule, and then extend rapidly up to the top of the photosphere with an amplitude maximun at $140 \mathrm{~km}$ (with our new altitude evaluation). Above this altitude, the velocity amplitude decreases in good agreement with previous results (Espagnet et al. 1995).

The velocities of the downflows at the edge of the exploding granules, are found between -0.2 and $-0.7 \mathrm{~km} \mathrm{~s}^{-1}$, depending on the granule. After the explosion, we observe in our example two columns of upwarding plasma (2 granules resulting from the explosion) which go right through the photosphere. As quoted above, a clear temperature reversal is seen above the separated velocity 


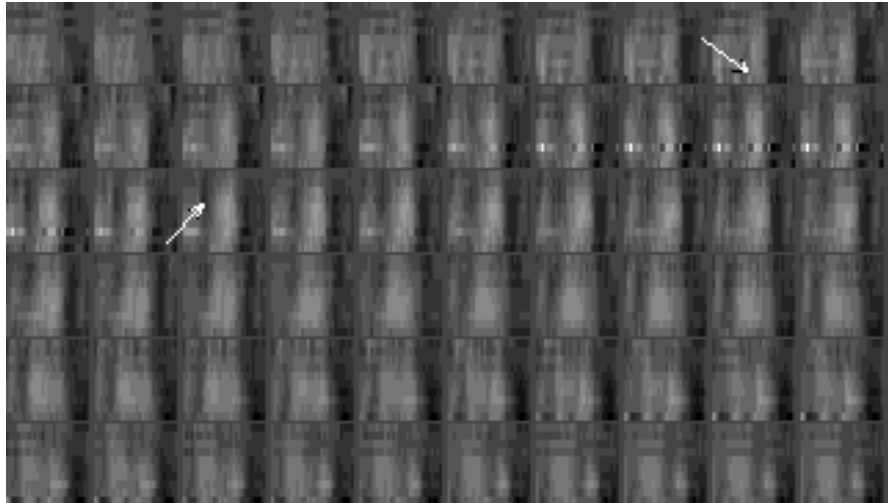

Fig. 5. Time sequence of Doppler velocity map in a vertical cross-section (slice) of the photosphere. White areas correspond to the upward velocity. The starting image of the sequence is located in the upper-left corner and the time evolution runs from the left to the right. The field of view in the $x$ direction is $5^{\prime \prime}$. The time step between two consecutive slices is $15 \mathrm{~s}$. The extrema of the Doppler velocities in this figure are -0.5 and $0.6 \mathrm{~km} \mathrm{~s}^{-1}$. The arrow in the upper-right shows the location of the Exploding Granule in the slice. The arrow in the left-middle part indicates the location of one of the Bright Plumes. The scale height has been magnified by a factor 7 with respect to the horizontal scale (see movie at http://mesola.obspm.fr/gallery/iv.mpg)

elements on each part of the exploding granule, like if we had a separation of the granule in two columns of plasma. These phenomena look like Rast's description (Rast 1995) where the maximun upflow is immediately adjacent to the maximun downflow. Unfortunately, our spatial resolution does not allow us to observe such details before the explosion.

\section{Scenario of the exploding granule through the photosphere}

The analysis of the observed properties and evolution of the exploding granules, enables us to draw a typical scenario of the time evolution (Fig. 6):

The plasma upflows in the first minutes ( 1 to 3 ), then in the following $2 \mathrm{~min}$ a generation of heating (bright plumes) occurs on the edges of the granules due to a braking action of the downflowing plasma , then a "connection" in temperature carries out between the intensity field of the granule and the "Bright Plume" during the following 2 to $3 \mathrm{~min}$. The granule explosion happens between 1 to 3 min after the intensity "connection". The division in intensity of the granule due to the explosion is clearly visible, and horizontal velocities of the elements resulting from the explosion are measured between 0.6 and $2 \mathrm{~km} \mathrm{~s}^{-1}$.

In the next two section sections we shall give the observational evidences for this scenario.

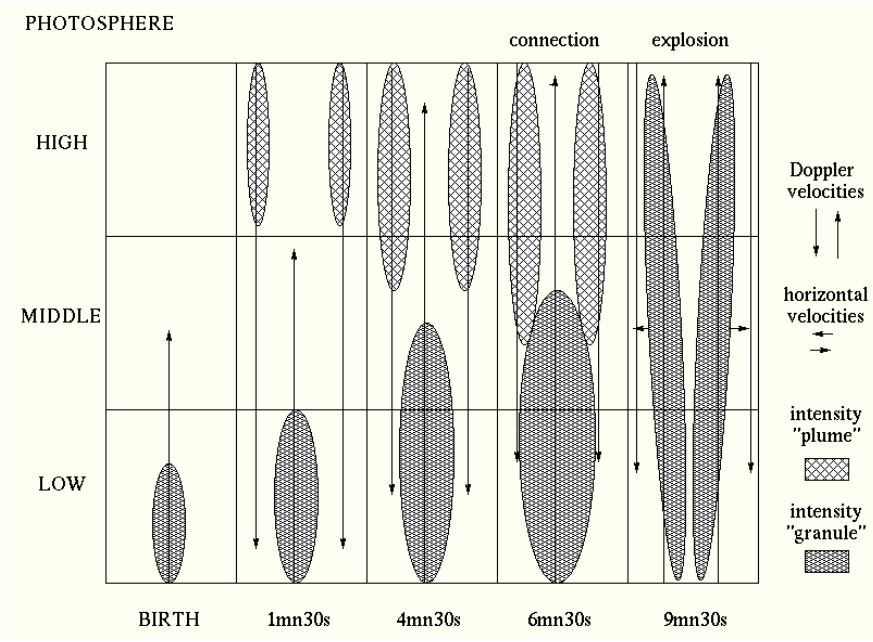

Fig. 6. Sketch illustrating the temporal evolution of intensity and velocity of an exploding granule through the photosphere

\section{Temporal evolution at the centre of the exploding granules}

In order to characterize the temporal evolution of the Doppler velocity and intensity field in the exploding granule, these quantities are plotted versus time (i.e. Figs. 7 and 9) at the centre of the exploding granules. To reduce the noise effects, the plotted quantities represent for each temporal step the average over the 8 closest pixels and the pixel at the centre of the exploding granules.

\subsection{Velocity}

The Doppler velocity, at the centre of the exploding granule, decreases in all the layers of the photosphere around the exploding time in Fig. 7.

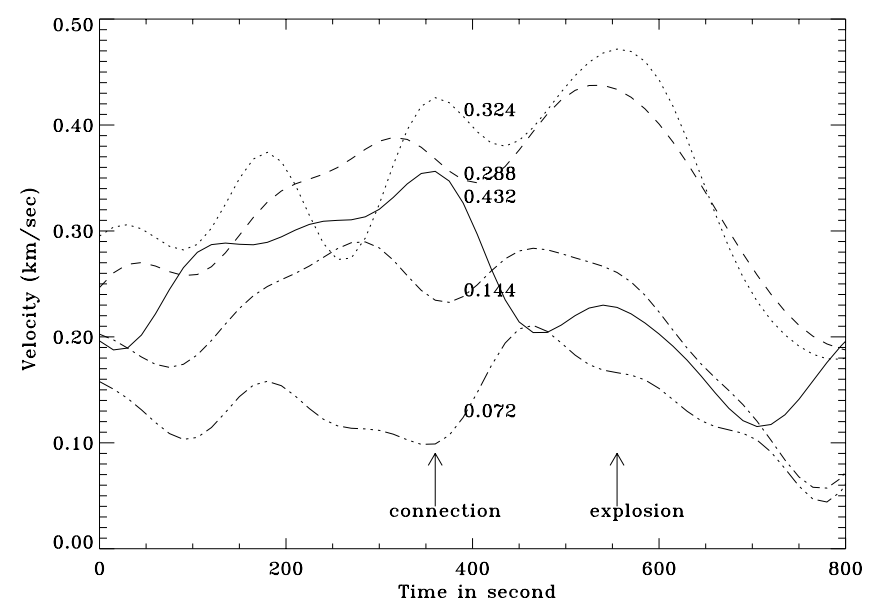

Fig. 7. Temporal evolution of the velocity $\left(\mathrm{km} \mathrm{s}^{-1}\right)$ at the centre of the exploding granules, at different depths in the photosphere 

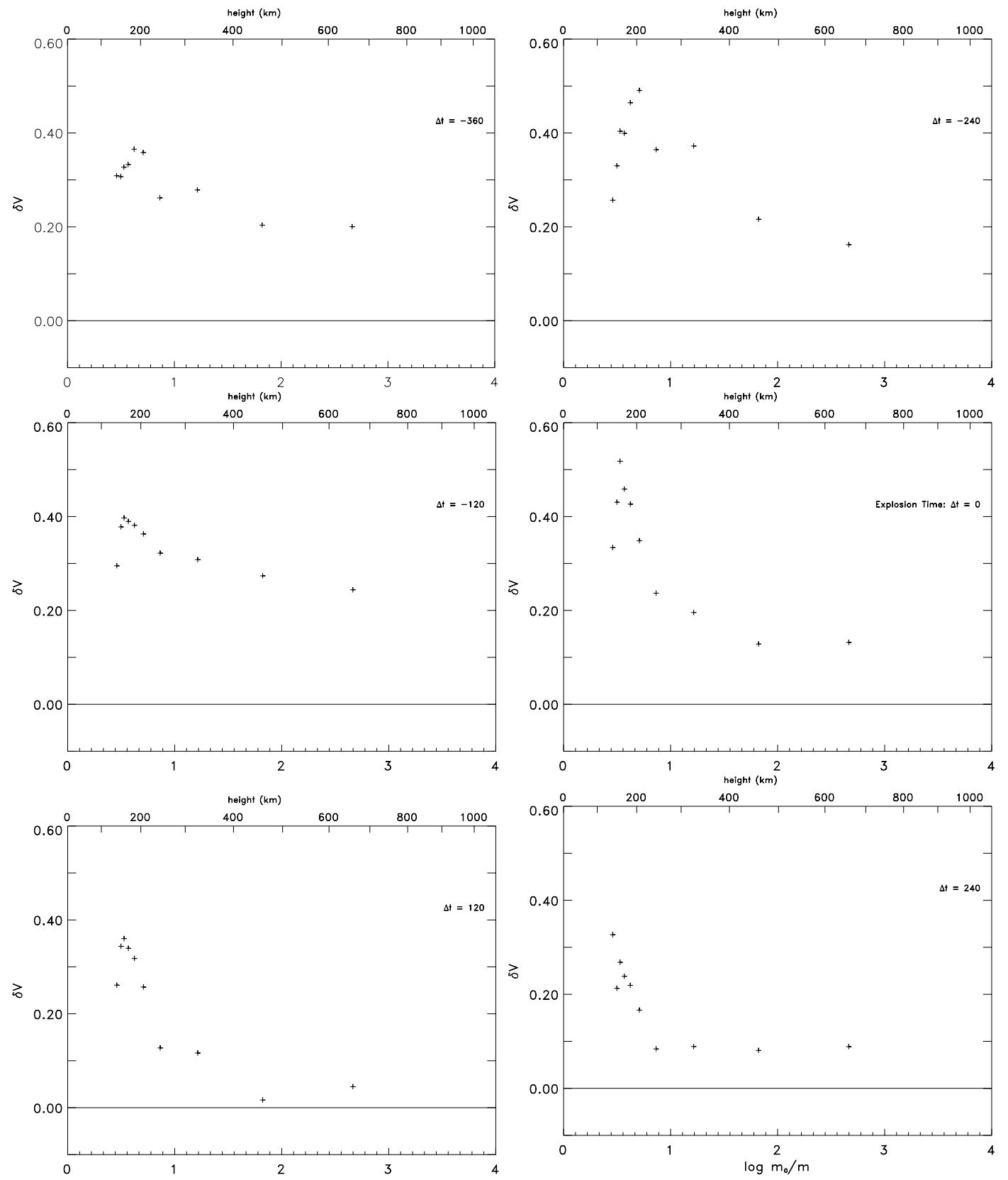

Fig. 8. $\delta V$ temporal evolution through the photosphere. Time is indicated as interval, in seconds, with respect to the explosion. The $x$-axis gives the photosphere height and the $\log \left(m_{0} / m\right)$ unit; the origin coincides at $h=0 \mathrm{~km}$ and unit standard optical depth

\subsection{Velocity fluctuations}

We note, in most cases of our sample, that the velocities at the top of the photosphere $(\Delta \lambda=0.072,0.144 \AA)$ are more or less constant around $0.2 \mathrm{~km} \mathrm{~s}^{-1}$. Although in the middle and low photosphere the velocity starts, in some cases, to decrease around the "connection" time, the top of the photosphere seems less sensitive to the explosion. The explosion time appears clearly in all of our sample as a key point of the quick modification (in less than a minute) of the velocity in the solar atmosphere.

In Fig. 7 , the velocity decreases after the explosion in all the atmosphere with a larger amplitude in the middle photosphere from $0.46 \mathrm{~km} \mathrm{~s}^{-1}$ to $0.18 \mathrm{~km} \mathrm{~s}^{-1}$. In our sample, this Doppler velocity decreases, at the maximum, from 0.8 to $0.1 \mathrm{~km} \mathrm{~s}^{-1}$ (sometimes to $-0.1 \mathrm{~km} \mathrm{~s}^{-1}$ ) but stays in general positive which indicates that the fluid is upwarding. Concerning the velocity downflow found on the edge of exploding granule (intergranule) up to $-0.7 \mathrm{~km} \mathrm{~s}^{-1}$, we do not think that the positive velocities found at the centre of granules are due to an effect of light diffusion. This result does not correspond to the downflow velocities expected by some authors (Rast 1995) with central plume formation with large downward velocities. 
The analysis of the observational data with the reponse functions (Fig. 2) allows us to determine velocity fluctuations at different heights in the photosphere. Generally, the tendency of a local maximum occurs around $h=150 \mathrm{~km}$ which probably indicates the location of the overshooting in the low photosphere.

This local maximun of $\delta V$ increases from $\sim 0.3$ to $0.5 \mathrm{kms}^{-1}$ at the explosion which is an increase of $70 \%$ over its initial value. Then the amplitude of the velocity fluctuations decreases with altitude Fig. 8.

During all the time sequence, before and after the explosion, $\delta V$ remains always positive in all the photosphere at the centre of the exploding granule. The decrease in altitude of $\delta V$ above the local maximum is sensitive to the explosion. The decrease of $\delta V$ with the altitude is sharp around the explosion and becomes flat after the explosion. The time interval during which the amplitude of $\delta V$ reduces by half with respect to its value at explosion lies between 60 and $390 \mathrm{~s}$, with a mean value of $210 \mathrm{~s}$ in our sample.

\subsection{Intensity fluctuations}

During the first minutes, in the low photosphere, we observe (in Fig. 9), the intensity growing phase at the centre of the exploding granule. The intensity generally decreases, at the centre of the exploding granule, before the "connection" time in the low and middle photosphere. For some of the exploding granules a shoulder and a steeper decrease is found around the explosion time in the low and middle photosphere.

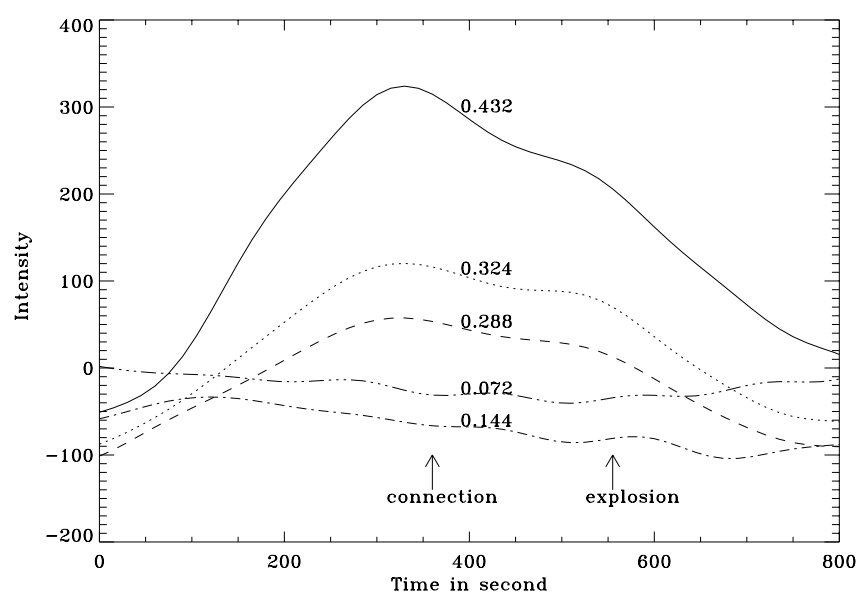

Fig. 9. Temporal evolution of the intensity at the centre of the exploding granules, at different depths in the photosphere

In the middle and high photosphere the negative values of the intensity fluctuations are characteristic of the temperature reversal ( $\Delta \lambda: 0.072$ and $0.144 \AA$ ). As the velocity behaviour, the intensity fluctuations in the middle and high photosphere seem less sensitive to the explosion event and are quite stable during our sequence.

\subsection{Temperature fluctuations}

The analysis of the observational data with response functions (Fig. 1) gives the opportunity to learn about the temperature fluctuations through the photosphere during the time sequence. Figure 10 shows the result of the predicted disturbances of temperature as a function of height. The selected spatial coordinates corresponds to the centre of the exploding granule 1 of our sample.

From the birth of the exploding granule, the temperature fluctuations have a maximum amplitude in the low photosphere which decreases linearly with altitude. The linear model appears acceptable for the temperature fluctuations in the low and middle photosphere, for all the exploding granules of our sample. Before the explosion $\delta T$ is positive in the low photosphere and negative in the middle and high photosphere. This is characteristic of the temperature reversal which occurs around the altitude of $150 \mathrm{~km}$. After the explosion $\delta T$ is negative in all the photosphere, in half cases of our sample. The slope of the linear fit in the low and middle photosphere, indicates the modifications of the temperature gradients in the photosphere during the exploding granule evolution. In our sample, the slope has the largest (or close to the largest) absolute amplitude (with negative value, see Fig. 10) before the explosion.

After the explosion the slope of the linear fit, decreases in amplitude and generally go to 0 (flat slope). We also note that after the explosion, the maximum $\delta T$ amplitude in the low photosphere reduces by half during the $3 \mathrm{mn}$ following the explosion.

\section{Temporal evolution at the centre of the Bright Plumes}

\subsection{Velocity}

The temporal evolution of the velocity at the location of the "Bright Plumes" are plotted in Fig. 11 at the same levels in the atmosphere as those used in Figs. 7 and 9.

Before the "connection", we observe, in the low photosphere, large downflows $\left(-0.3\right.$ and $-0.7 \mathrm{~km} \mathrm{~s}^{-1}$ in all our sample) which then slow down till the explosion time, up to $0 \mathrm{~km} \mathrm{~s}^{-1}$. The downflow velocities are more vigourous in the low photosphere compared to the high photosphere. In these higher layers, we observe a weakening or a the steadyness of the velocities, depending on the Bright Plume case. This scenario is common to all the observed "Bright Plumes". This looks like a braking action of the plasma, coming from the granule, due to the small size of the intergranular region. This braking is certainly related to mass conservation of the flow.

\subsection{Intensity}

The temporal evolution of the intensity at the location of the "Bright Plumes" is plotted in Fig. 12 at the same levels in the atmosphere as those used in Figs. 7 and 9 for exploding granules. 

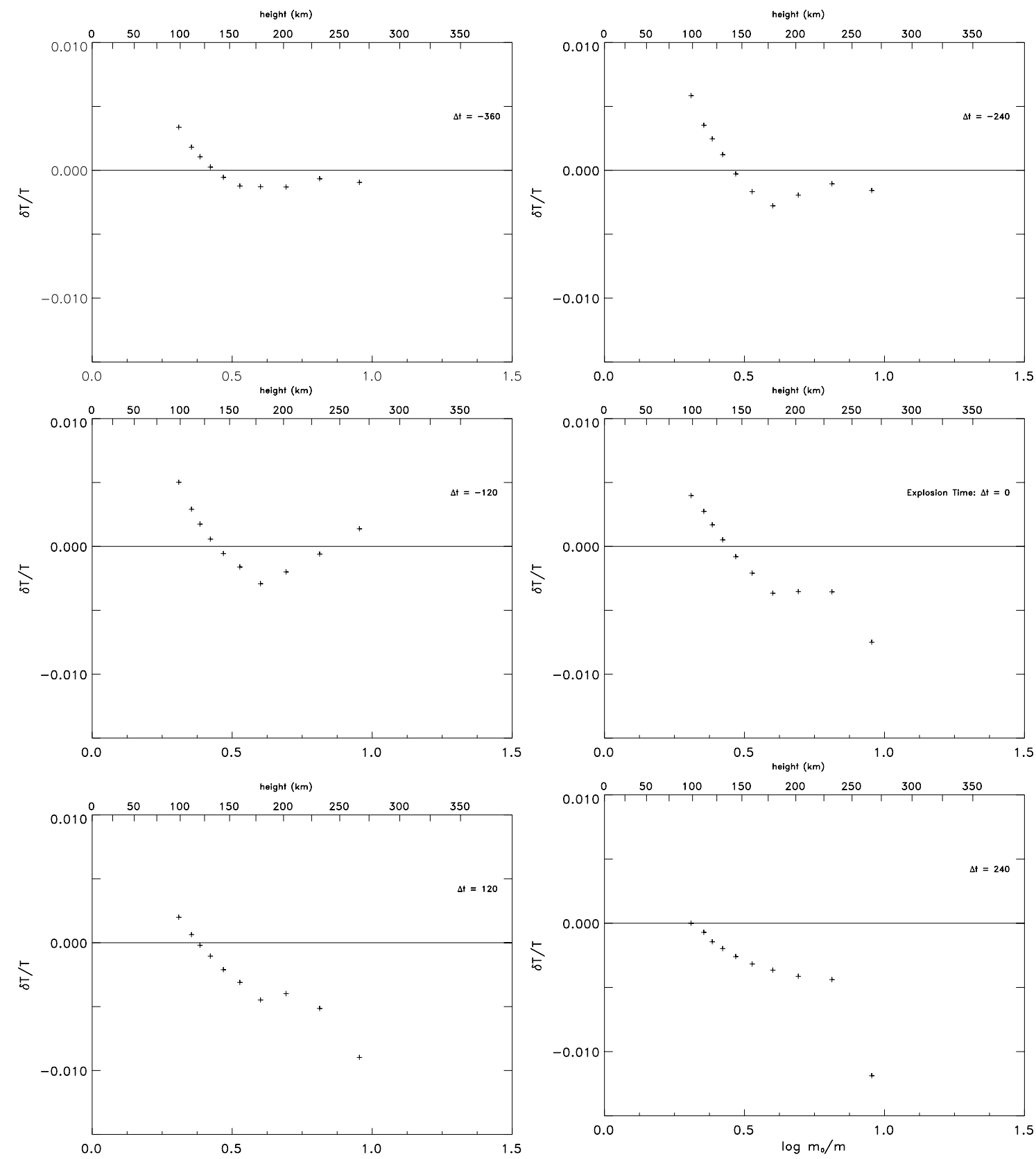

Fig. 10. Same as Fig. 8 but for temperature fluctuations

At the beginning, in the low photosphere $(\Delta \lambda=0.432$ and $0.324 \AA$ ), negative intensity values are observed although in the overlapping layers the intensity is positive indicating the "Bright plume" formation. Then, intensity increases with time up to the explosion in low layers of the photosphere. At this level, the "connection" is visible as a shoulder of the slope. In the middle and high photosphere the increase goes up to the "connection" time and then decreases. In this example, the "connection" appears more preponderant in the intensity change in the upper layers. This is more or less observed in the half part of our samples. The fact that there is no brightness counterpart at the bottom of the photosphere below the "Bright Plumes", indicates what there is no direct convective origin of the heat in this phenomenon.

\section{Discussion and conclusions}

This paper presents, for the first time, the temporal evolution of exploding granules in intensity and Doppler velocity through the solar photosphere. A particular attention in the data reduction has been paid to minimize the perturbations due to the seeing and the 5-min oscillations. Temperature and velocity response functions for $\mathrm{NaD}_{2}$ line have been computed to probe the atmosphere at different levels: low, middle and high photosphere.

From our analysis, the origin of the brightness of these "Plumes" is related to the strength of the downflow. These brightnesses appear around the granule where the downward velocities are the most intense. They are more or less located isotropically around the granule, depending 


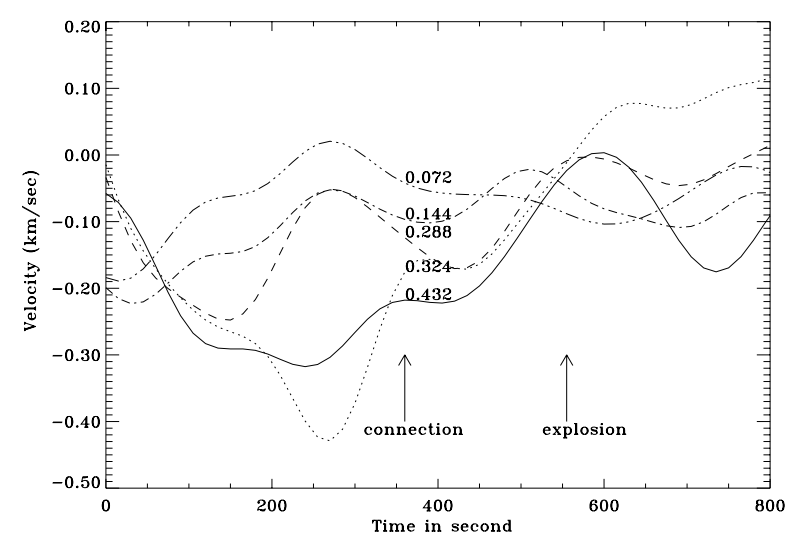

Fig. 11. Temporal evolution of the velocity $\left(\mathrm{kms}^{-1}\right)$ in the centre of the Bright Plume, at different depths in the photosphere

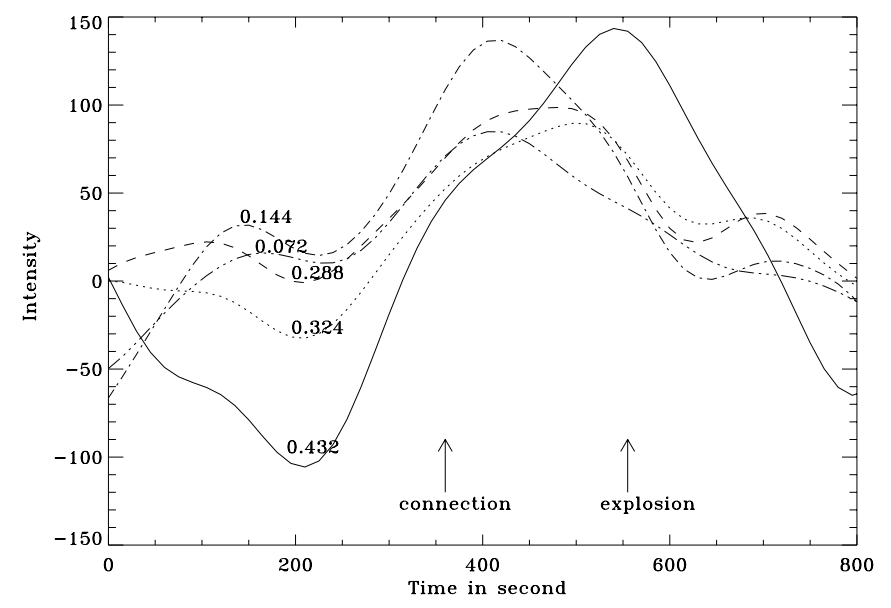

Fig. 12. Temporal evolution of the intensity in the centre of the Bright Plume, at different depths in the photosphere

on the flow properties of the exploding granule. For some exploding granules the brightnesses are found only on one side where the downflows are very important. Below the "Bright Plumes", which are located in the high and middle photosphere, there is no bright intensity counterpart which indicates no direct convective origin of these brightness.

During the "Bright Plumes" event, the downflow are less and less vigourous, indicating like a braking of the plasma. The relation between the braking and the brightness does not seem to be due to a shock because the highest velocities in the middle photosphere are subsonic; $0.7 \mathrm{~km} \mathrm{~s}^{-1}$ represents at this levels only $11 \%$ of the sound velocity.

Regarding now the elements of the granule resulting from the explosion, we find that the intensity (cf. temperature) reversal was located above the elements which have the largest ascending velocities (above $0.4 \mathrm{~km} \mathrm{~s}^{-1}$ ).

In this temporal sequence, we find a singular phenomenon which is an intensity bridge between the Bright plumes (high and middle photosphere) and the granule in the low photosphere. This kind of junction seems temporally related, in two third of our sample, to the generation of the explosion. The explosion happens 1 to 3 min after the "connection" phenomenon.

Up to now, we do not know which kind of physical mechanism produces this increase of temperature in the high photosphere in the intergranular lane. It could be related to the shock waves produced by the supersonic motions near the upper thermal boundary layer in the border of exploding granule (Cattaneo et al. 1990; Ludwig 2000).

The velocity measured at the centre of the exploding granule during its evolution in the photosphere, remains mostly positive (upflow) even after the explosion which is quite different from what is generally expected by different models (Rast 1995) except the one described by Nelson \& Musman (1978) where the continuum of the central darkness is formed at a lower temperature, and compatible with upward motions. Light diffusion effect does not seem to produce this upflow because velocities up to $-0.7 \mathrm{~km} \mathrm{~s}^{-1}$ are found on the edges of the exploding granule where velocity gradients are strong.

The scenario sketched out in Fig. 6 for the evolution of an exploding granule through the photosphere, summarized the different observed steps which lead to the explosion.

However, we cannot definitively establish, with this small sample, the causality relation between the "connection" and the explosion. It is certainly important and necessary to confirm (or disprove), this result with higher spatial resolution observations and with a large number of explosive events. Simultaneous observations of the chromosphere to learn about the origin of this phenomenon in altitude could also be necessary. This would allow us to learn about the nature of this "connection": waves or other phenomena?

Acknowledgements. We wish to thank Ch. Coutard, R. Hellier, F. Colson and the Pic du Midi Staff for their technical assistance during this work. The photometric measurements were made with the microdensitometer "M.A.M.A." of the Institut National des Sciences de L'Univers in Paris. This work was supported by the Centre National de la Recherche Scientifique (C.N.R.S., UMR 5572) and the Programme National Soleil Terre (P.N.S.T.).

\section{References}

Brandt, P., Ferguson, S., Scharmer, G., et al. 1991, A\&A, 241, 219

Carlier, A., Chauveau, F., Hugon, M., \& Rosch, J. 1968, C. R. Acad. Sc. Paris, 266, 119

Carlsson 1986, Upsalla Astronomical Observatory, Report No. 33

Cattaneo, F., Hurlburt, N. E., \& Toomre, J. 1990, ApJ, 349, 63

Eibe, M. et al., 2000, in preparation

Espagnet, O., Muller, R., Roudier, T., Mein, N., \& P., P. M. 1995, 109, 79

Hirzberger, J., Bonet, J. A., Vázquez, M., \& Hanslmeier, A. 1999, ApJ, 527, 405 
Karpinsky, V. N. 1989, in Solar Photosphere: Structure, Convection, and Magnetic Fields: Proceedings of the 138th Symposium of the International Astronomical Union, held in Kiev, U.S.S.R., May 15-20, 1989. International Astronomical Union. Symposium, No. 138, ed. Jan Olof Stenflo (Kluwer Academic Publishers, Dordrecht), 67

Ludwig, H. G. 2000, private communication

Nelson, G. \& Musman, S. 1978, ApJ, 222, 69

Nesis, A., Mattig, W., \& Durrant, C. J. 1988, A\&A, 201, 153

Ploner, S., Solanki, S., \& Gadun, A. 1999, ApJ, 352, 679

Rast, M. 1995, ApJ, 443, 863
Rieutord, M., Roudier, T., Malherbe, J. M., \& Rincon, F. 2000, A\&A, 357, 1063

Roudier, T., Mein, P., Muller, R., et al. 1991, A\&A, 248, 237

Stein, R. F., \& Nordlund, A. 1998, ApJ, 499, 914

Title, A. M., Tarbell, T. D., Topka, K. P., et al. 1989, ApJ, 336,475

Topka, T., \& Title, A. 1991, in Solar Interior and Atmosphere, ed. A. N. Cox, W. C. Livingston, \& M. S. Matthews (Univ. of Arizona Press), 727

Vernazza, J., Avrett, E., \& Loeser, R. 1981, ApJ, 45, 635

Wiehr, E. \& Kneer, F. 1988, A\&A, 195, 310 